\title{
Avaliação Ultrassonográfica Intracoronária da Carga Aterosclerótica no Tronco da Artéria Coronária Esquerda Prediz a Extensão da Doença no Restante da Árvore Coronária: Análise Piloto
}

Breno de Alencar Araripe Falcão', Gustavo Rique Morais ${ }^{1}$, João Luiz de Alencar Araripe Falcão', Rafael Cavalcante Silva', Expedito Eustáquio Ribeiro', Eulógio Emílio Martinez¹, Pedro Alves Lemos

\section{RESUMO}

Introdução: A imagem de placas ateroscleróticas em território extracardíaco, por meio do ultrassom das carótidas, tem sido proposta como forma indireta para estimar o risco coronário. Considerou-se a hipótese de que a avaliação isolada do tronco da artéria coronária esquerda (TCE) pudesse predizer diretamente a carga aterosclerótica no restante da árvore coronária. Métodos: O estudo incluiu pacientes com diagnóstico de doença arterial coronária com indicação de intervenção coronária percutânea (ICP), que apresentassem TCE sem estenose significativa. Durante a ICP, realizou-se ultrassom intracoronário (USIC) do TCE e das três artérias coronárias em que foram mensuradas as áreas luminal, da membrana elástica externa e da placa e a carga de placa em cada vaso. Resultados: Foram incluídos 17 pacientes com média de idade de $56 \pm 8,9$ anos, $71 \%$ do sexo masculino e $41 \%$ diabéticos. Apesar de nenhum paciente apresentar estenose significativa no TCE à angiografia ou ao ultrassom, observou-se, no TCE, evidência de aterosclerose com área de placa de $8,4 \pm 2,7 \mathrm{~mm}^{2}$ e carga de placa de $33,5 \pm 8,9 \%$. Correlação significativa entre o TCE e o restante das coronárias foi observada para todos os parâmetros ultrassonográficos avaliados: área luminal $(r=0,5)$, área da membrana elástica externa $(r=0,7)$, área de placa $(r=0,6)$ e carga de placa $(r=0,4)$. Conclusões: Nesta análise piloto, a carga aterosclerótica coronária global pôde ser estimada a partir da avaliação isolada do TCE por USIC.
ABSTRACT

Intravascular Ultrasound Assessment of Atherosclerotic Plaque Burden at the Left Main Coronary Artery Predicts the Severity of Coronary Artery Disease: A Pilot Study

Background: Non-cardiac atherosclerotic imaging, including carotid ultrasound, has been proposed as an indirect way to estimate coronary risk. The authors hypothesize that the isolated assessment of the left main coronary artery (LMCA) could predict the atherosclerotic burden in the remainder of the coronary tree. Methods: The study included patients with a diagnosis of coronary artery disease with an indication for percutaneous coronary intervention $(\mathrm{PCI})$, without significant LMCA stenosis. During PCI, LMCA and three-vessel intravascular ultrasound (IVUS) examination was performed and the lumen area, external elastic membrane area, plaque area, and plaque burden were measured. Results: A total of 17 patients were included with mean age of $56 \pm 8.9$ years, $71 \%$ male, and $41 \%$ diabetics. Although none of the patients had LMCA stenosis by angiography or IVUS, evidence of atherosclerosis was observed with a plaque area of $8.4 \pm 2.7 \mathrm{~mm}^{2}$ and plaque burden of $33.5 \pm 8.9 \%$. A significant correlation between the LMCA and the rest of the coronary arteries was observed for all IVUS parameters: lumen area $(r=0.5)$, external elastic membrane area $(r=0.7)$; plaque area $(r=0.6)$, and plaque burden $(r=0.4)$.

1 Instituto do Coração do Hospital das Clínicas da Faculdade de Medicina da Universidade de São Paulo (InCor/HCFMUSP) - São Paulo, SP, Brasil.

Correspondência: Breno de Alencar Araripe Falcão. Rua Oscar Freire, 1.753/41 B - Cerqueira César - São Paulo, SP, Brasil - CEP 05409-011 E-mail: breno.falcao@incor.usp.br

Recebido em: 12/6/2011 • Aceito em: 21/8/2011 
Essa associação é relevante para a construção de escores de predição de risco coronário, como marcador substituto da carga de aterosclerose global da árvore coronária.

DESCRITORES: Doença da artéria coronária. Ultrassonografia. Placa aterosclerótica.

A placa aterosclerótica coronária representa o substrato patológico fundamental para a ocorrência de eventos isquêmicos cardiovasculares. ${ }^{1}$ Estudos de necropsia demonstraram presença de aterosclerose coronária mais extensa em vítimas de morte súbita, comparativamente àqueles com morte de causa não-cardíaca. ${ }^{2,3}$ Recentemente, uma análise conjunta de dados de seis estudos de ultrassom intravascular seriados demonstrou relação direta entre a carga aterosclerótica coronária global e sua progressão e o risco para eventos adversos cardiovasculares maiores, em especial para a necessidade de revascularização miocárdica. ${ }^{4}$

A quantificação da aterosclerose em outros leitos vasculares, como a espessura da íntima-média nas carótidas, já é amplamente utilizada na avaliação do risco coronário e sua incorporação na estratificação de risco permitiu melhorar a capacidade discriminatória do risco estimado pelo escore de risco de Framingham. ${ }^{5,6} \mathrm{~A}$ utilização de estimativas diretas, por métodos de imagem, da carga aterosclerótica coronária global em escores de estratificação de risco cardiovascular tem potencial para aprimorar a capacidade discriminatória desses escores, orientando estratégias preventivas e terapêuticas mais adequadas.

O tronco da artéria coronária esquerda é um segmento de grande importância clínica, que apresenta particularidades anatômicas, como localização proximal, grande calibre e comprimento curto, que facilitam sua avaliação por meio de métodos de imagem invasivos e não-invasivos. Neste projeto piloto é analisada a hipótese de que a avaliação isolada por imagem do tronco da artéria coronária esquerda pudesse predizer a carga aterosclerótica no restante da árvore coronária, refletindo a carga aterosclerótica global coronária, em indivíduos com doença arterial coronária previamente diagnosticada.

\section{MÉTODOS}

Os critérios de inclusão compreenderam indivíduos portadores de doença arterial coronária com indicação clínica de intervenção coronária percutânea (ICP) que apresentassem tronco da artéria coronária esquerda sem estenose significativa à angiografia e que preenchessem pelo menos uma das seguintes características de maior risco para novos eventos coronários: padrão coronário multiarterial (pelo menos dois vasos com obstrução an-
Conclusions: In this pilot study, the coronary atherosclerotic burden could be estimated from the isolated assessment of the LMCA by IVUS. Such an association is relevant for the development of future coronary risk scores, as a surrogate marker of the global coronary tree atherosclerotic burden.

KEY-WORDS: Coronary artery disease. Ultrasonography. Plaque, atherosclerotic.

giográfica > 50\% em diâmetro), diabetes melito (diagnóstico prévio da doença em uso de hipoglicemiantes orais ou de insulina ou recém-diagnosticado por meio de dosagem de glicemia de jejum > 126 mg/dl aferida em duas ocasiões distintas) ou síndrome coronária aguda recente (há menos de 14 dias).

Foram excluídos pacientes em fase aguda de infarto do miocárdio e aqueles com antecedente de cirurgia de revascularização miocárdica, instabilidade hemodinâmica, fração de ejeção do ventrículo esquerdo $<30 \%$, creatinina sérica $>2 \mathrm{mg} / \mathrm{dl}$ ou alergia a contraste iodado. Também foram excluídos pacientes cujo ultrassom intracoronário (USIC) foi considerado de qualidade inadequada para análise.

Durante o procedimento de ICP, os pacientes incluídos foram submetidos a USIC do tronco da artéria coronária esquerda e de pelo menos um dos três ramos arteriais coronários, sendo preferencialmente obtidas imagens de todos os ramos epicárdicos principais, quando tecnicamente possível. As médias dos parâmetros ultrassonográficos de área luminal, área da membrana elástica externa, área de placa (área da lâmina elástica externa menos a área luminal) e carga de placa (razão da área de placa e da área da membrana elástica externa x 100) foram mensuradas para o tronco da artéria coronária esquerda e para o restante da árvore coronária, a fim de se avaliar a correlação entre esses parâmetros (Figura 1).

As imagens ultrassonográficas foram obtidas com o cateter Eagle Eye Gold (Volcano Therapeutics, Rancho Cordova, Estados Unidos), de $20 \mathrm{MHz}$ e 2,9 F de diâmetro, utilizando o console In Vision Gold (Volcano Therapeutics, Rancho Cordova, Estados Unidos). Antes da aquisição das imagens, era administrada nitroglicerina intracoronária (100-200 mcg) e realizados subtração do ringdown (artefato de imagem na proximidade do cateter) e ajustes nas imagens em escala de cinza de modo a melhorar o contraste da imagem e facilitar e interpretação do exame. A seguir, o transdutor do cateter de ultrassom era posicionado pelo menos $5 \mathrm{~cm}$ distal ao óstio da artéria que se desejava avaliar e realizava-se o recuo automático do cateter à velocidade de $0,5 \mathrm{~mm} / \mathrm{s}$, com o auxílio do dispositivo R100 (Volcano Therapeutics, Rancho Cordova, Estados Unidos). A análise das imagens ultrassonográficas foi realizada off-line com o programa PcVH2.2. 
Todos os pacientes receberam informações sobre os procedimentos, concordaram em participar e assinaram termo de consentimento livre e esclarecido, previamente aprovado pelas comissões de ética do Instituto do Coração do Hospital das Clínicas da Faculdade de Medicina da Universidade de São Paulo (InCor/ HCFMUSP - São Paulo, SP, Brasil).

As variáveis categóricas foram descritas por suas contagens e frequências e as variáveis contínuas foram expressas por média e desvio padrão. Para análise de correlação utilizou-se o teste de Pearson. Considerou-se como significativo $\mathrm{P}<0,05$.

\section{RESULTADOS}

O estudo incluiu, no total, 17 pacientes, com média de idade de $56 \pm 8,9$ anos e $71 \%$ do sexo masculino. De modo geral, o perfil clínico era de alto risco, sendo $41 \%$ diabéticos, $41 \%$ portadores de doença multiarterial e 53\% com síndrome coronária aguda recente (Tabela 1). Além do tronco da coronária esquerda ( $n=17), 42$ outras artérias coronárias principais foram analisadas (artéria descendente anterior $=15$; artéria circunflexa $=12$; artéria coronária direita $=13$; outros ramos importantes $=2$ ). A extensão total analisada de tronco da coronária es-

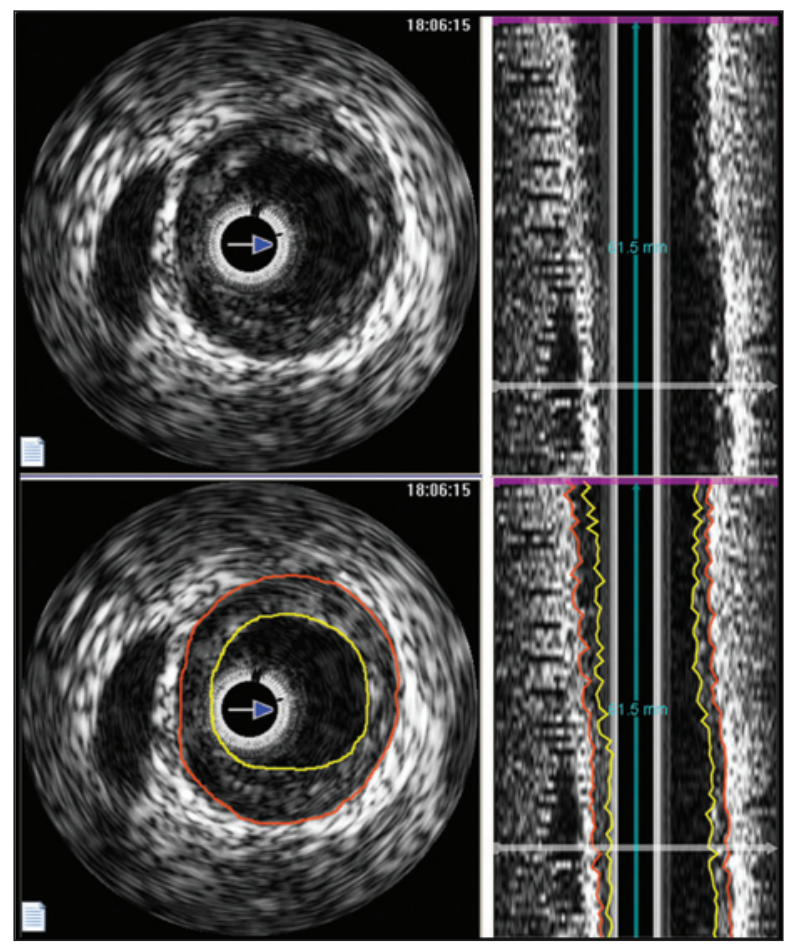

Figura 1 - À esquerda, corte ultrassonográfico transversal da artéria coronária após aquisição da imagem (painel superior) e após análise (painel inferior), com delimitação da área luminal (traçado central), da membrana elástica externa (traçado externo) e da placa aterosclerótica (região entre os traçados). À direita, corte longitudinal da artéria após aquisição da imagem (painel superior) e após análise (painel inferior), com delimitação análoga de traçados. querda foi de 106,8 $\mathrm{mm}$ (média de 6,3 \pm 6,1 $\mathrm{mm}$ ), enquanto a extensão total das demais artérias foi de 2.106,4 mm (média de 50,2 \pm 8,7 mm).

A despeito da ausência de obstruções luminais significativas no tronco da artéria coronária esquerda, observou-se evidência clara de aterosclerose nesse segmento, com área de placa de 8,4 $\pm 2,7 \mathrm{~mm}^{2}$ e carga de placa de 33,5 $\pm 8,9 \%$ (Tabela 2 ). Houve correlação significativa entre o tronco da artéria coronária esquerda e o restante das artérias coronárias para todos os seguintes parâmetros ultrassonográficos avaliados: área luminal $(r=0,5)$, área da membrana elástica externa $(r=0,7)$, área de placa $(r=0,6)$ e carga de placa $(r=0,4)$ (Tabela 3) (Figura 2).

\section{DISCUSSÃO}

O principal achado deste estudo piloto foi a determinação da existência de correlação significativa entre o grau de doença aterosclerótica presente no tronco da artéria coronária esquerda e o acometimento do restante da árvore coronária ao USIC (Figura 3). Tais resultados sugerem um potencial para utilização de medidas de imagens diretas da aterosclerose no tronco da artéria coronária esquerda como marcador substituto da carga de aterosclerose total da árvore coronária, que, por sua vez, se correlaciona com a ocorrência de eventos adversos cardiovasculares. ${ }^{4}$

TABELA 1

Características da população

\begin{tabular}{lc} 
Características clínicas & \\
\hline Idade*, anos & $56 \pm 8,9$ \\
Sexo masculino, \% & 71 \\
Diabetes melito, \% & 41 \\
Hipertensão arterial sistêmica, \% & 65 \\
Dislipidemia, \% & 59 \\
Tabagismo, \% & 29 \\
Síndrome coronária aguda recente, \% & 53 \\
Padrão coronário multiarterial, \% & 41 \\
Uso prévio de estatina, \% & 53 \\
\hline Características laboratoriais & \\
\hline Colesterol total*, mg/dl & $163,2 \pm 33,8$ \\
LDL-colesterol*, mg/dl & $104,6 \pm 28,3$ \\
Triglicérides*, mg/dl & $105,2 \pm 37,1$ \\
HDL-colesterol*, mg/dl & $37,6 \pm 12,1$ \\
Glicemia de jejum*, mg/dl & $123,1 \pm 56,3$ \\
Hemoglobina glicada*, \% & $6,2 \pm 1,1$ \\
Creatinina*, mg/dl & $1 \pm 0,2$ \\
\hline
\end{tabular}

* Valores expressos como média \pm desvio padrão.

HDL-colesterol = colesterol de lipoproteína de alta densidade; LDL-colesterol = colesterol de lipoproteína de baixa densidade. 
TABELA 2

Parâmetros ultrassonográficos do tronco da artéria coronária esquerda e das artérias coronárias avaliadas

\begin{tabular}{lcc}
\hline & $\begin{array}{c}\text { Tronco da artéria coronária esquerda } \\
(\mathbf{n}=\mathbf{1 7})\end{array}$ & $\begin{array}{c}\text { Demais coronárias } \\
(\mathbf{n}=\mathbf{4 2})\end{array}$ \\
\hline Área luminal*, $\mathrm{mm}^{2}$ & $16,9 \pm 5,1$ & $8,3 \pm 2,1$ \\
Área da MEE* $\mathrm{mm}^{2}$ & $25,3 \pm 6,7$ & $14,6 \pm 3,7$ \\
Área de placa*, $\mathrm{mm}^{2}$ & $8,4 \pm 2,7$ & $6,3 \pm 2,2$ \\
Carga de placa*, \% & $33,5 \pm 8,9$ & $42,1 \pm 6,7$ \\
\hline
\end{tabular}

* Valores expressos como média \pm desvio padrão.

MEE $=$ membrana elástica externa; $\mathrm{n}=$ número de pacientes.

TABELA 3

Correlação entre parâmetros ultrassonográficos do tronco da artéria coronária esquerda e das artérias coronárias avaliadas

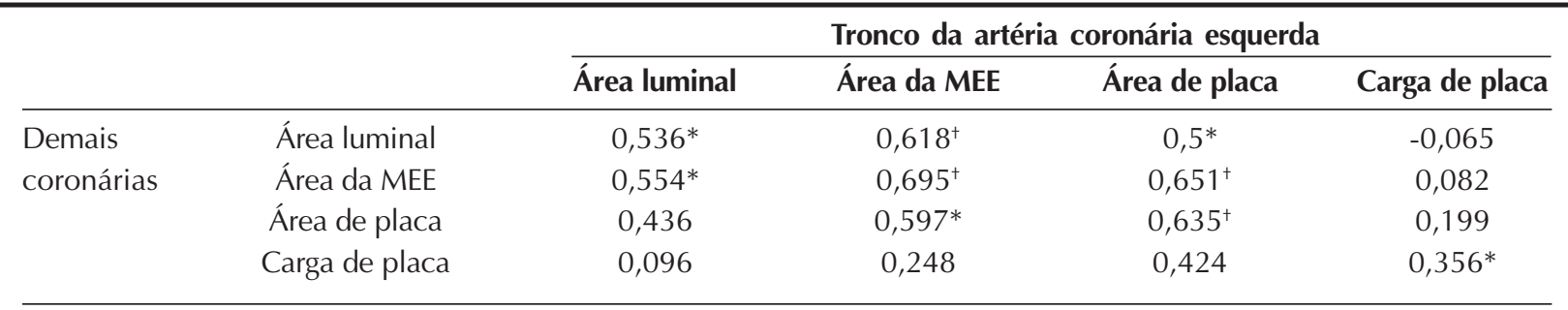

Valores nas células referem-se ao coeficiente de correlação de Pearson.

$* \mathrm{P}<0,05 ;{ }^{+} \mathrm{P}<0,01$.

MEE = membrana elástica externa.

Estudos com ultrassom intravascular seriados demonstraram correlação da progressão ou regressão da aterosclerose no tronco da artéria coronária esquerda com o nível de colesterol de lipoproteína de baixa densidade (LDL-colesterol), independentemente da idade do paciente. ${ }^{7-9}$ Além dessa associação com fatores de risco tradicionais, observou-se correlação positiva independente entre a progressão de placa no tronco da artéria coronária esquerda e o nível de lipoproteína (a) [Lp(a)], um dos novos preditores de risco cardiovascular. ${ }^{10} \mathrm{~A}$ extensão da progressão da aterosclerose no tronco da artéria coronária esquerda também se relacionou de forma positiva e linear com o risco de eventos clínicos cardiovasculares derivados de três escores de risco estabelecidos para prevenção primária. ${ }^{11}$

Esses dados em conjunto suportam a utilização de medidas da aterosclerose no tronco da artéria coronária esquerda para composição de novos índices preditores de risco cardiovascular, potencialmente com maior capacidade discriminatória na estratificação do risco. Apesar de o USIC do tronco da artéria coronária esquerda ser um exame seguro, com baixas taxas de complicações e de oferecer medidas acuradas da aterosclerose nesse vaso, trata-se de um procedimento invasivo e de alto custo, limitando sua utilização ampla como ferramenta para rastreamento de doença e para

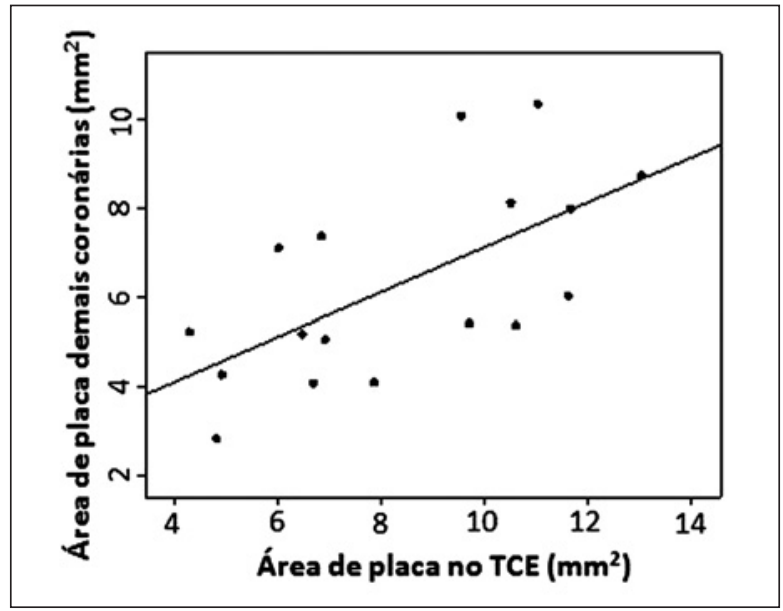

Figura 2 - Gráfico representativo da correlação entre a área média de placa no tronco da artéria coronária esquerda e a área média de placa nas demais artérias coronárias como um todo, avaliadas ao ultrassom $(r=0,635 ; P=0,006)$. TCE $=$ tronco da artéria coronária esquerda.

composição de escores de risco para prevenção primária. Nesse sentido, a angiotomografia computadorizada das artérias coronárias poderia se tornar uma ferramenta promissora. ${ }^{12}$ 


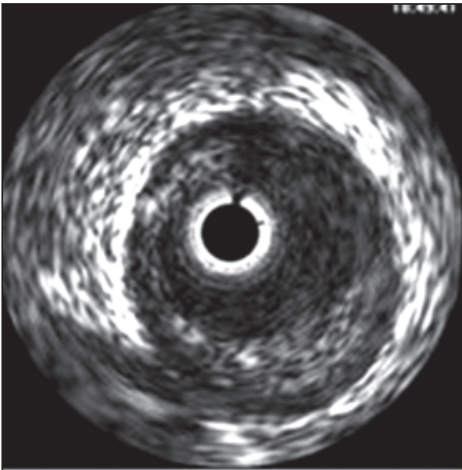

TCE

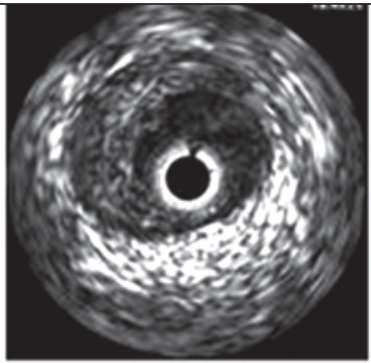

DA

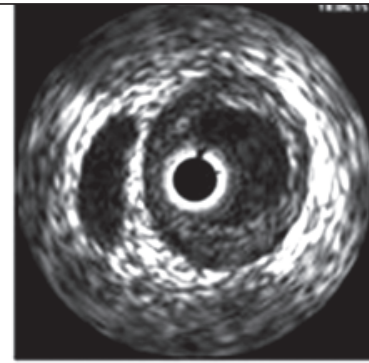

CX

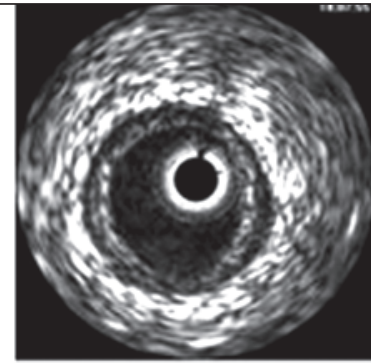

CD

Figura 3 - Imagens ilustrativas da concordância do grau de acometimento aterosclerótico nos principais ramos arteriais de um mesmo paciente. A figura mostra cortes transversais de ultrassom intravascular do tronco da artéria coronária esquerda e das três principais artérias coronárias do mesmo paciente. $\mathrm{CD}=$ artéria coronária direita; $\mathrm{CX}=$ artéria circunflexa; $\mathrm{DA}=$ artéria descendente anterior; TCE $=$ tronco da artéria coronária esquerda.

Ainda em relação à transposição desses dados para prevenção primária, é importante observar que, no presente trabalho, a população incluída foi composta por portadores de doença arterial coronária com perfil clínico de alto risco para novos eventos cardiovasculares, de modo que a propriedade de a aterosclerose no tronco da artéria coronária esquerda refletir a carga aterosclerótica na árvore coronária total em uma população de menor risco, no contexto da prevenção primária, precisaria ser explorada.

\section{CONCLUSÕES}

A carga aterosclerótica global da árvore coronária pôde ser estimada a partir da avaliação isolada do tronco da artéria coronária esquerda por meio do USIC. Essa associação é relevante como ferramenta para construção de índices ateroscleróticos de predição de risco cardiovascular, utilizando sistemas de imagem coronária invasivos e não-invasivos.

\section{SUPORTE FINANCEIRO}

Este trabalho contou com o apoio da Fundação de Amparo à Pesquisa do Estado de São Paulo (FAPESP).

\section{CONFLITO DE INTERESSES}

Os autores declaram não haver conflito de interesses relacionado a este manuscrito.

\section{REFERÊNCIAS}

1. Vlodaver Z, French R, van Tassel RA, Edwards JE. Correlation of the antemortem coronary angiogram and the postmortem specimen. Circulation. 1973;47(1):162-9.

2. Roberts WC. Diffuse extent of coronary atherosclerosis in fatal coronary artery disease. Am J Cardiol. 1990;65(12):2F-6F

3. Cliff WJ, Heathcote CR, Moss NS, Reichenbach DD. The coronary arteries in cases of cardiac and noncardiac sudden death. Am J Pathol. 1988;132(2):319-29.

4. Nicholls SJ, Hsu A, Wolski K, Hu B, Bayturan O, Lavoie A, et al. Intravascular ultrasound-derived measures of coronary atherosclerotic plaque burden and clinical outcomes. J Am Coll Cardiol. 2010;55(21):2399-407.

5. Lorenz MW, Markus HS, Bots ML, Rosvall M, Sitzer M. Prediction of clinical cardiovascular events with carotid intima-media thickness: a systematic review and meta-analysis. Circulation. 2007;115(4):459-67.

6. Polak JF, Pencina MJ, Pencina KM, O'Donnell CJ, Wolf PA, D'Agostino RB. Carotid-wall intima-media thickness and cardiovascular events. N Engl J Med. 2011;365(3):213-21.

7. von Birgelen C, Hartmann M, Mintz GS, Baumgart D, Schmermund A, Erbel R. Relation between progression and regression of atherosclerotic left main coronary artery disease and serum cholesterol levels as assessed with serial long-term (> 12 months) follow-up intravascular ultrasound. Circulation. 2003;108(22):2757-62.

8. Hong MK, Lee CW, Kim YH, Park DW, Lee SW, Park CB, et al. Usefulness of follow-up low-density lipoprotein cholesterol level as an independent predictor of changes of coronary atherosclerotic plaque size as determined by intravascular ultrasound analysis after statin (atorvastatin or simvastatin) therapy. Am J Cardiol. 2006;98(7):866-70.

9. Hartmann M, von Birgelen C, Mintz GS, van Houwelinger GK, Eggebrecht $H$, Böse D, et al. Relation between plaque progression an low-density lipoprotein cholesterol during aging as assessed with serial long-term (> 12 months) followup intravascular ultrasound of the left main coronary artery. Am J Cardiol. 2006;98(11):1419-23.

10. Hartmann M, von Birgelen C, Mintz GS, Stoel MG, Eggebrecht $\mathrm{H}$, Wieneke $\mathrm{H}$, et al. Relation between lipoprotein (a) and fibrinogen and serial intravascular ultrasound plaque progression in the left main coronary arteries. J Am Coll Cardiol. 2006;48(3): 446-52.

11. von Birgelen $C$, Hartmann M, Mintz GS, van Houwelingen G, Deppermann N, Schmermund A, et al. Relationship between cardiovascular risk as predicted by established risk scores versus plaque progression as measured by serial intravascular ultrasound in left main coronary arteries. Circulation. 2004; 110(12):1579-85.

12. Falcão JLAA, Gurudevan SV, Shiozaki AA, Coelho Filho OR, Falcão BAA, Sales FJR, et al. Associação entre a densidade radiológica da placa à tomografia de coronárias com 64 colunas de detectores e a composição da placa ao ultrassom intravascular com técnica de histologia virtual: resultados de um comparação pareada prospectiva. Rev Bras Cardiol Invasiva. 2009;17(3):327-34. 